\title{
From the symbolic analysis of virtual faces to a smiles machine
}

\author{
Magalie Ochs ${ }^{1}$, Edwin Diday ${ }^{2}$, and Filipe Afonso $^{3}$ \\ ${ }^{1}$ CNRS LTCI Télécom ParisTech, \\ magalie.ochs@telecom-paristech.fr \\ 2 CEREMADE Université Paris Dauphine, \\ diday@ceremade.dauphine.fr \\ ${ }^{3}$ SYROKKO - Aéropôle Paris / Roissy CDG \\ afonso@syrokko.com
}

\begin{abstract}
In this paper, we present an application of symbolic data processing for the design of virtual character's smiling facial expressions. A collected database of virtual character's smiles directly created by users has been explored using symbolic data analysis methods. An unsupervised analysis has enabled us to identify the morphological and dynamic characteristics of different types of smiles as well as of combinations of smiles. Based on the symbolic data analysis, to generate different smiling faces, we have developed procedures to automatically reconstitute smiling virtual faces from a point in a multidimensional space corresponding to a Principal Component Analysis (PCA) plane.
\end{abstract}

Index Terms-Smiles, Symbolic data processing, Virtual characters, Graphical tool

\section{INTRODUCTION}

Nowadays, computers are more and more used to endow roles typically embodied by humans, such as a tutor in a virtual learning class or an assistant for a virtual task realization. Such roles are often embodied by animated cartoons or human like virtual characters, called Embodied Conversational Agent (ECA) [1], able to communicate through verbal and non-verbal behavior with the user. Research has shown that when people are involved in an interaction with such virtual characters, they tend to react naturally and socially as they would do with another person [2].

One important social cue during social interactions is a smile [3]. Smiles may enhance the global perception of a person [4]. Moreover, a smiling virtual character enhances the human-machine interaction, for instance the perception of the task, of the agent, and the motivation and enthusiasm of the user [5], [6].

A smile is one of the simplest and most easily recognized facial expressions. To create a smile, the two muscles zygomatic majors, on either side of the face, have to be activated. However, others muscles may be implied in an expression of a smile. Moreover, a smile may have several meanings - such as amusement and politeness - depending on subtle differences in the characteristics of the smile itself and of other elements of the face that are displayed with the smile.

In this paper, we present research work that is aimed at creating a repertoire of smiles to give the capability to an embodied conversational agent to express different types of smiles during an interaction with a user. The existing methods to construct repertoires of virtual characters' facial expressions are presented in the next section (Section II). In our approach, to identify the facial expressions associated with different smiles, a corpus of virtual character's smiles directly created by users (presented in Section III) has been analyzed with descriptive methods of symbolic analysis (Section IV). An unsupervised analysis of the data has enabled us to identify types and combinations of smiles (Section V). Based on this analysis, procedures have been proposed to automatically generate different smiles continuously in a Principal Component Analysis (PCA) plane (Section VI). We conclude with Section VII.

\section{State of ART}

Symbolic data processing has been used in several application domains. Indeed, people are not only interested in the study of individuals but also in the identification of classes of individuals. Symbolic data analysis has been used for instance for recommendations in marketing [7], for medical applications [8], in biology to study pigs illnesses [9], in text mining [10], etc. However, as far as we know, symbolic data analysis methods have never been explored for virtual character's facial expressions synthesis.

In the domain of virtual characters, several computational models have been proposed to give the capability to virtual characters to display emotions or feelings, in particular through their facial expressions [11], [12]. Indeed, evaluation studies have shown that virtual characters expressing emotions enhance human-machine interaction [13], [14].

A facial expression arises from muscular contractions. To animate the facial expression of a virtual character, both the morphological and dynamic characteristics of the virtual face should be considered. To give the capability to a virtual character to express through its face particular emotional states and communicative intentions, the latter should be endowed with a lexicon, i.e., a dictionary of facial expressions linking morphological and dynamic characteristics of the face to specific meanings. To create a repertoire of a virtual character's facial expressions, the method that is commonly used consists of exploiting the empirical and theoretical studies in psychology that have highlighted the morphological and 
dynamic characteristics of human's facial expressions. In particular, most of the computational models are based on the "Big six" theory of Ekman and Friesen [15] describing the facial expressions associated with six emotions (happy, fear, anger, surprise, disgust, and sadness). To allow virtual characters to display a large number of emotional expressions, a dimensional approach was also proposed [16]. In the dimensional models, a new expression is often created by applying some arithmetical operations, such as linear interpolation, on numerical definitions of discrete emotions placed in a multidimensional space.

In the present work, we adopt a dimensional approach to create a repertoire of virtual character's expressions. However, the proposed method differs from existing models on different points. First of all, in the existing dimensional models, each facial expression is represented by a vector of continuous values. In our work, we represent a facial expression by discrete variables. Indeed, in the literature in human and social sciences [15], [17], [18], [19], the characteristics of emotional facial expressions, and in particular of smiling facial expressions, are not generally described with precise intensity of the muscles contractions. Consequently, in our model, the facial expressions are represented by discrete variables (with values such as "high", "low"). In this context, the symbolic data analysis offers methods particularly suitable to such data. Moreover, contrary to most of computational models, we do not focus on emotions but on the specific signal of smiles.

Last but not least, to collect data, we adopt a particular method in which the users directly created the virtual character's smiles. This method has several advantages. First, it considers directly the user's perception of the virtual character instead of replicating findings of human's non-verbal behavior on virtual characters. Moreover, a corpus of virtual character's facial expressions avoids the problem of acted human's expressions (not necessarily similar to spontaneous expressions) or the difficulty to collect spontaneous expressions (i.e. , to induce the emotional state to the actor). Secondly, this method may generate one-to-many correspondences between socioemotional states and facial expressions: the virtual characters have not one unique smile but may display several smiles to express a same state. The platform to collect the virtual agent's smiles is described in more detail in the next section.

\section{A VIRTUAL SMILING FACES DATABASE}

To construct a database of smiling virtual faces, a first problem consists of identifying how to represent the different smiles and their characteristics.

The most common type of smile is the amused smile (also called felt or Duchenne smile). Another type, which is often thought of as the amused smile's opposite is the polite smile (also called non-Duchenne, false, social smile). A specific smile appears in the facial expression of embarrassment [17]. In this paper, we focus on the smiles of amusement, politeness and embarrassment. These smiles have the advantage to have been explored in the human and social sciences literature both from the encoder point of view (from the point of view of the person who smiles) [17], [18] and from the decoder point of

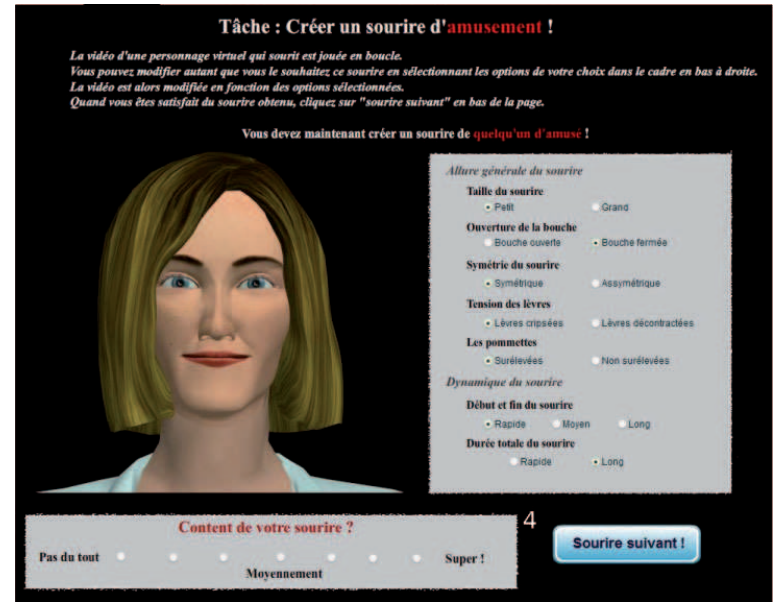

Fig. 1. Screenshot of the web application to create smiles.

view (from the point of view of the one who perceived the smile) [19].

The amused, polite and embarrassed smiles are distinguishable by their distinct morphological and dynamic characteristics. Although no consensus exists on the morphological and dynamic characteristics of these smiles, the researchers in human and social sciences generally agree on the characteristics to consider to describe the different smiles: the amplitude of the smile, its duration, the openness of the mouth, the symmetry of the lip corner, the tension of the lips, the velocity of the onset and offset of the smile, and the cheek raising [17], [18].

In order to identify the morphological and dynamic characteristics of the amused, polite, and embarrassed smiles of a virtual character, we have proposed a user-perceptive approach: we have created a web application that enables a user to easily create different types of smiles on a virtual character's face (Figure 1). Through radio buttons on an interface, the user could generate any smile by choosing a combination of seven parameters (amplitude of smile, duration of the smile, mouth opening, symmetry of the lip corner, lip press, the velocity of the onset and offset of the smile, and cheek raising). We have considered two or three discrete values for each of these parameters (for instance, small or large for the amplitude of the smile). When the user changes the value of one of the parameters, the corresponding video of a virtual character smiling is automatically played. The user was instructed to create one animation for each type of smile. The user had to indicate his satisfaction related to the smile he has created (through a Likert scale of 7 points). As described in the following, this value of satisfaction has been used to oversample the data.

Three hundred and forty eight participants (with 195 females) with a mean age of 30 years have created smiles. We then collected 1044 descriptions of smiles: 348 descriptions for each smile type (amused, polite and embarrassed). On average, the participants were satisfied by the created smiles (5.28 on 
a Likert scale of 7 points) ${ }^{1}$ (for more details on the collected smiles, see [20]).

The user indicated a level of satisfaction for each created smile (a level that varied between 1 and 7). The level of satisfaction was used to oversample the data with the assumption that smiles with a high level of satisfaction were more reliable than smiles with a low level. To give a higher weight to the smiles with a high level of satisfaction, we did oversampling $^{2}$ such as: each created smile was duplicated $n$ times, where $n$ is the level of satisfaction associated with this smile. So, a smile with a level of satisfaction of 7 is duplicated 7 times whereas a smile with a level of satisfaction of 1 is not duplicated. The resulting data set is composed of 5517 descriptions of smiles: 2057 amused smiles, 1675 polite smiles, and 1785 embarrassed smiles.

Finally, in the database, each created smile is represented by 9 variables:

- an identification number $(I D \in[1,5517])$,

- a smile type (TYPE $\in\{$ amused,polite, embarrassed $\})$,

- the size of the smile (SMILE_SIZE $\in\{$ small,large $\}$ ),

- the openness of the mouth (MOUTH_OPEN $\in$ $\{$ open, close $\}$ ),

- the symmetry of the smile (SMILE_SYMETRIE $\in$ $\{$ sym., asym\}),

- the lips tension (LIPS_TENSION $\in\{$ no tension, tension\}),

- the cheek raising (SMILE_AU6 $\in\{n o-A U 6, A U 6\}$ ),

- the onset and offset of the smile (SMILE_ONOFFSET $\in\{$ short, medium, long $\}$ ),

- the total duration of the smile (SMILE_DURATION $\in$ $\{$ short, long $\}$ ).

The discrete values of the variables correspond to those indicated by the user in the application of smile creation (Figure 1).

To analyze the data, the variables have been handled as symbolic variables corresponding to diagram of frequencies of the discrete values (i.e. the modalities). In the next section, we describe more precisely the database with symbolic analysis methods.

\section{Symbolic ANALYSis OF POLITE, EMBARRASSED AND AMUSED SMILES}

A symbolic analysis [21], [22], [23], [24] is based on a characterization of each class by the variations of the variables describing the class. For qualitative variables, the variation is represented through a diagram of the frequencies, corresponding to "symbolic data". In our context, the symbolic data are represented by the diagram of frequencies of the values of each variable describing a smile (the smile type, the openness of the smile, its size, the symmetry of the smile, the lips tension, the cheek raising, the onset and offset of the smile,

\footnotetext{
${ }^{1}$ Globally, the users' satisfaction was similar for the three smiles (between 5.2 and 5.5)

${ }^{2}$ Note that an undersampling could be also applied by not considering smiles with a low level of satisfaction. We have given priority to oversampling to have the maximum of smile descriptions to model the variability of smile expressions.
}

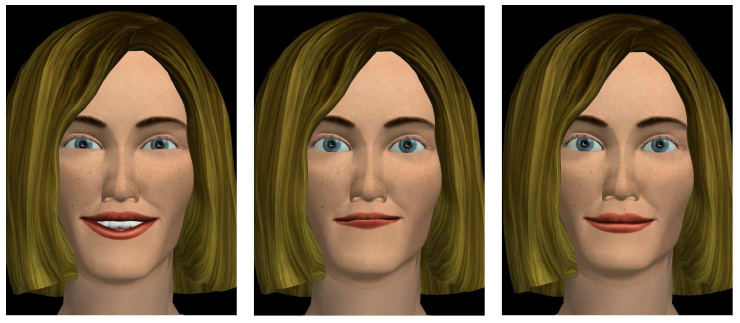

Fig. 3. Images of the three smiles (at the apex) with the most frequent characteristics identified in the database (from the left to the right) : amused smile, embarrassed smile and polite smile.

and the total duration of the smile). In the next paragraph, we describe the symbolic data.

\section{A. Frequencies of the smiles' characteristics}

To illustrate the frequency of each discrete value of the variables for each type of smile, a graphic representation with a 3D star has been used (Figure 2). This graphical representation has been generated with the SOE method [25] implemented in the freeware SODAS. An European consortium of 15 universities and companies has produced this academic freeware SODAS ${ }^{3}$. The module SOE of this software is for graphical visualization of symbolic data.

In Figure 2, each axis represents a variable characterizing smiles: tension of the lips (LIPS_TENSION), the cheek raising (SMILE_AU6) duration of the onset and offset (SMILE_ONOFFSET), total duration of the smile (SMILE_DURATION), smile size (SMILE_SIZE), openness of the mouth (MOUTH_OPEN) and the symmetry of the smile (SMILE_SYMETRIE). The star (Figure 2) regroups the data for each concept, i.e., for each smile type (amused, polite, embarrassed) represented by a particular color.

This graphical representation shows that the amused smiles are mainly characterized by an absence of lips tension (92.2\%), a long duration $(84.4 \%)$, a large size $(83.6 \%)$, an openess of the mouth $(85.6 \%)$ and a cheek raising $(78.4 \%)$. Even though the difference is less significant, the amused smiles seem more often described with a symmetry (59.9\%). The embarrassed smiles are characterized by a lips tension (74.6\%), a small size $(73.1 \%)$, a closed mouth $(81.8 \%)$, an assymmetry of the smile $(59.1 \%)$ and an absence of cheek raising $(59 \%)$. The duration of these smiles seems more frequently long $(56.4 \%)$. The smiles of politeness have both the characteristics of the amused smiles and of the embarrassed smiles. Indeed, they are characterized by an absence of lips tension (69.4\%), a small size $(67.7 \%)$, a close mouth $(76 \%)$, a symmetry of the smile $(67.1 \%)$ and the absence of cheek raising $(58.9 \%)$. The three smiles with the predominant characteristics are illustrated in Figure 3.

\section{B. The discriminant variables of smiles}

In order to identify the relevant variables to distinguish the types of smiles, a ranking of the variables ordered by their

\footnotetext{
${ }^{3}$ http://www.info.fundp.ac.be/asso/sodaslink.htm
} 


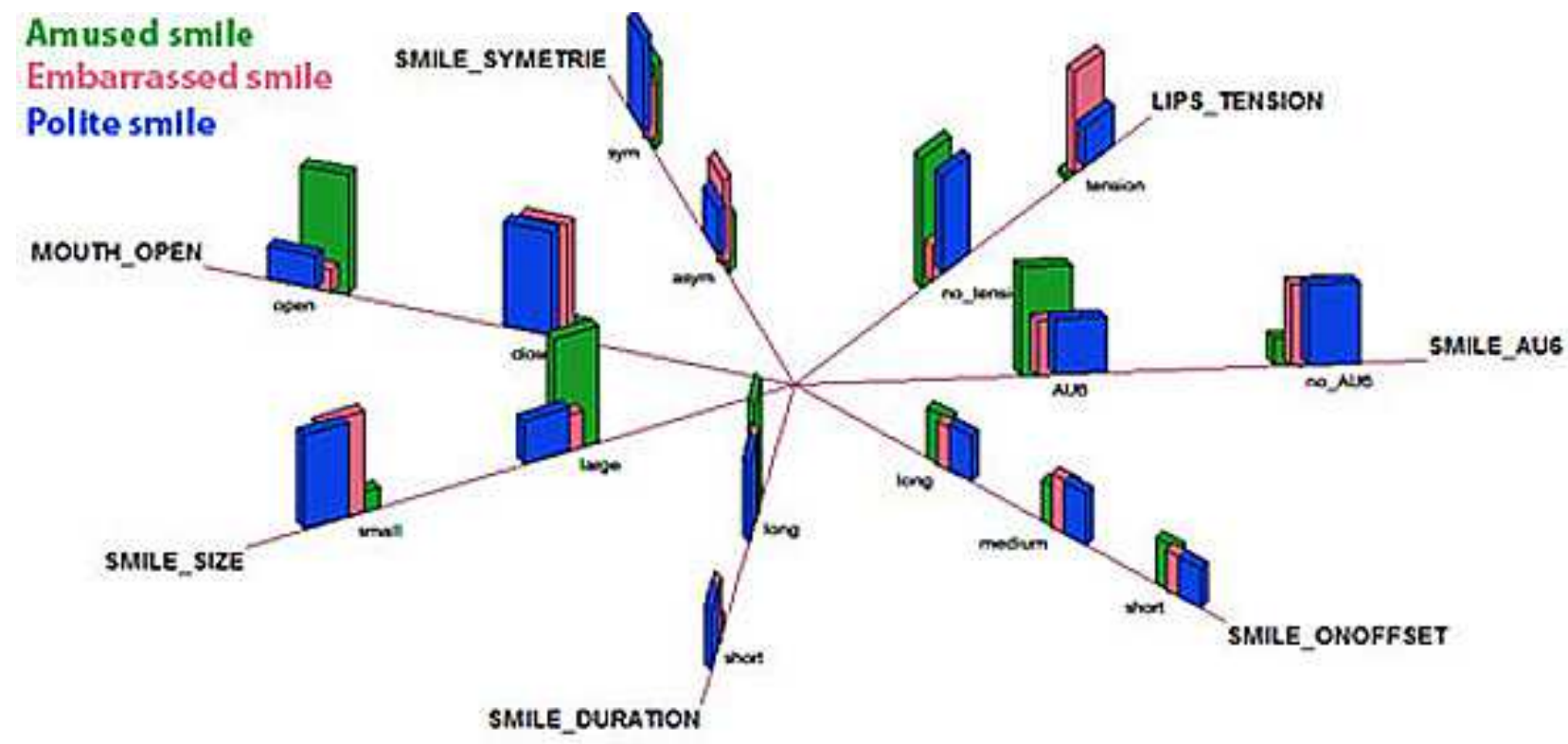

Fig. 2. ZOOM star of the morphological and dynamic characteristics of the amused, embarrassed and polite smile.

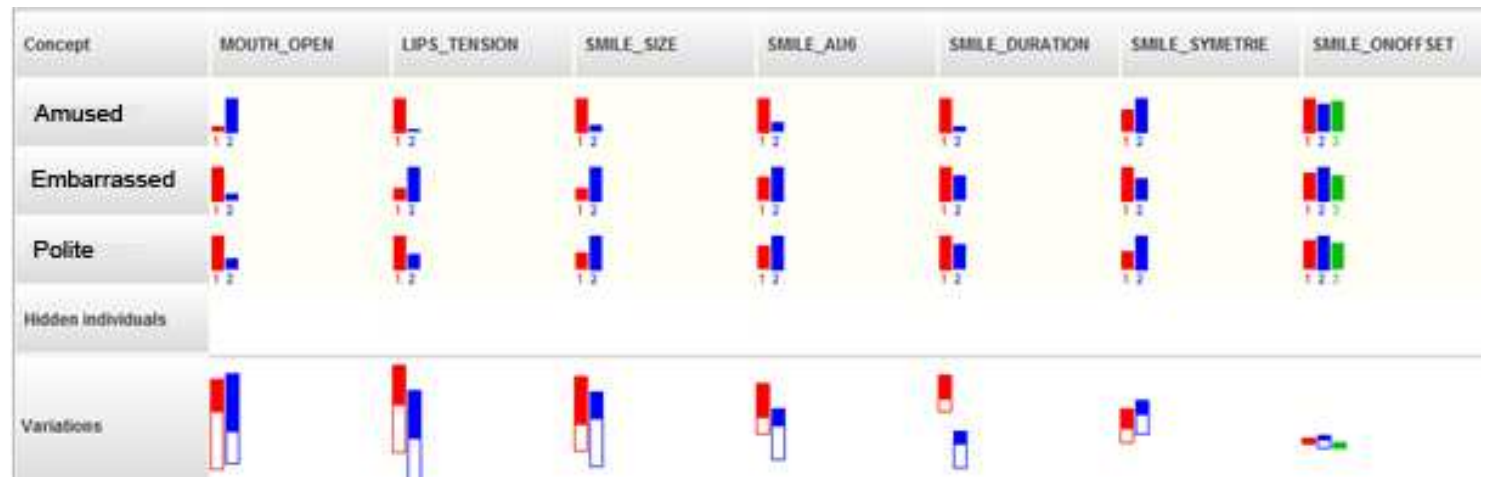

Fig. 4. Descriptives variables of the smiles ordered by the discriminant power (ascending order).

discriminant power (by ascending order, Figure 4) for each type of smile has been generated with the module TABSYR of the software SYR ${ }^{4}$.

The openness of the mouth (MOUTH_OPEN) appears as the most discriminant variable among the 7 variables describing the smiles. This variable discriminates in particular the smiles of amusement compared to the smiles of politeness and embarrassment. The variable of the lips tension (LIPS_TENSION) is also important to distinguish between the amused and polite smiles and the embarrassed smiles. The less discriminant variables, i.e., the size of the smile (SMILE_SIZE) and the cheek raising (SMILE_AU6), make it possible to distinguish between the amused smiles and the polite and embarrassed smiles. Even if the symmetry of the smile (SMILE_SYMETRIE) has smaller discriminant power, it discriminates the embarrassed smiles compared to the polite and amused smiles. The variable describing the onset and the offset (SMILE_ONOFFSET) does not appear as essential to distinguish the three types of smiles.

\footnotetext{
${ }^{4}$ This software is provided by SYROKKO company.
}

\section{UNSUPERVISED SYMBOLIC ANALYSIS OF SMILES}

In the previous section, we made the assumption that only three types of smiles appear in the database: the smile of amusement, politeness, and embarrassment. In this section, our objective is to explore, with an unsupervised symbolic analysis, the different types of smiles that can be distinguished without the assumption of the existence a priori of three types of smiles. For this purpose, we have used a dynamic clustering algorithm with the module NETSYR of the software SYR. This software enables the computation of the PCA of a symbolic data table in which the rows represent each class and the columns are the modalities of the histogram. In other words, the symbolic data table is considered as a numeric data table in which each cell contains the frequency of the modality of one symbolic variable for a given class.

We have studied the partitions of 3 to 15 classes. The choice of the number of classes has been done empirically by testing the partition until 15 classes. The curve of the inertias is illustrated in Figure 5.

There are different mathematical criteria to choose the appropriate number of classes. For example, in [26], 30 


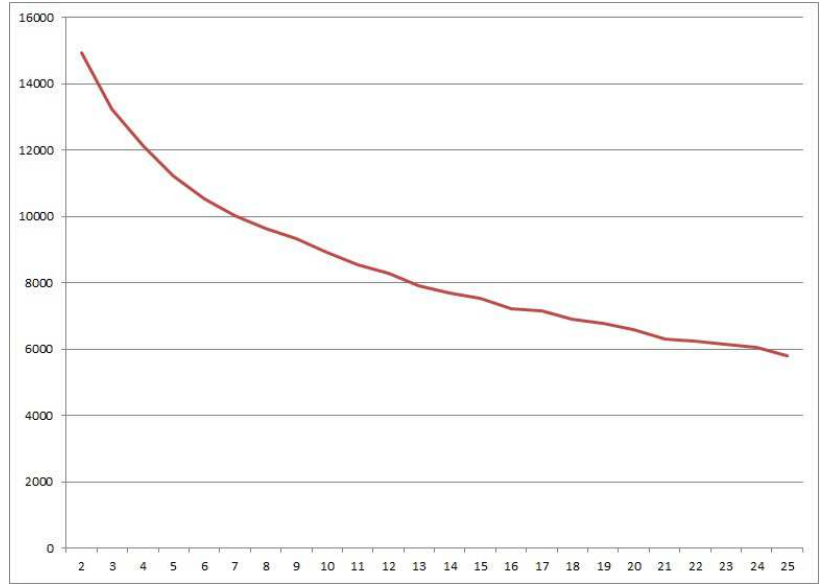

Fig. 5. Curve of the inertias for the partitions from 2 to 25 classes.

procedures are compared by Monte Carlo simulation. In [27], the authors compare the Calinsky and Harabasz, I-index, Cindex, Gamma_Index, the Beale Test and others based on the Poisson law. They give an extension of some criteria to symbolic data. The conclusion is that the indexes give different results as they express different aspects of the cluster. In this paper, we have used the sum of the intra-cluster inertia. Of course, there is not one unique solution. Consequently, the choice of the best mathematical criterion may be subjective. In our context, the choice, of 15 classes, is based on the decision of the expert on virtual smiles. This choice is supported by the curve of inertias (Figure 5) showing an inflexion between the partitions of 14 and 17 classes. We present in the following section $\mathrm{B}$, the partition in 15 classes.

\section{A. Typology of smiles in three classes}

The first unsupervised classification in 3 classes enables us to analyze if, in fact, the 3 classes of smile predefined $a$ priori (amusement, politeness and embarrassment) exist for the considered variables describing smiles (Figure 2). Regarding this unsupervised analysis, we have considered all the variables describing the smiles, except the variable corresponding to the type of smile (SMILE_TYPE) to avoid a presupposition on the existence a priori of the 3 smile types. The partition is illustrated in Figure 6.

Regarding the types of smiles (SMILE_TYPE), this typology in 3 classes shows that the class C3 is of type 1 (amused smile), the class $\mathrm{C} 2$ is of type 3 (polite smile) and the class $\mathrm{C} 1$ is of type 2 (embarrassed smile). Whereas the class C3 is mainly composed of amused smiles, the classes $\mathrm{C} 2$ and $\mathrm{C} 1$ correspond more particularly to a combination of polite and embarrassed smiles. Finally, this typology with 3 classes does not correspond exactly to the typology defined a priori. Indeed, there is no class only composed of embarrassed or polite smiles. These two smiles seem to be mixed. Consequently, two new classes appear in the typology corresponding to a combination of polite and embarrassed smiles with a predominance of one of the two types.

The optimized criterion for this classification is the inertia rate. The inertia rate for the classification in 3 classes following the 3 modalities of SMILE_TYPE (supervised classification) is 16051 . The inertia rate for the classification in 3 classes presented above (unsupervised classification) is 13235 . This unsupervised classification contains classes more homogeneous than in the supervised classification.

\section{B. Typology of smiles in 3 to 15 classes}

To refine this typology of smiles, we have studied the partitions of 3 to 15 classes. In order to consider all the classes resulting from the partitions of 3 to 15 classes, we have computed a PCA on the prototypes associated with the classes of all the partitions of 3 to 15 classes, i.e., we have considered the prototypes of the 117 classes. The prototypes are the means of the histograms of the class. Then, we have represented the 15 classes, themselves clustered in 5 classes. Figure 7 illustrates the partition on 15 classes by the representation of the 15 associated prototypes and by the representation of a partition in 5 classes of these 15 prototypes obtained by the same algorithm (i.e., dynamic clustering).

This unsupervised analysis shows partitions with a high frequency of prototypic smiles: the class named "Amused", "Embarrassed", and "Polite" (Figure 7) that have the main characteristics of the prototypic smiles described in Section IV (Figure 1). The other classes highlight different types of combinations of smiles: the class named "Embarrassed-Polite" and "Amused-Polite". The smiles of politeness appear often in combination with other smiles (embarrassed or amused). In contrast, the smiles of embarrassment are rarely combined with the smiles of amusement. These results are coherent with the meanings conveyed by these smiles, embarrassment and amusement being relatively opposed since it is difficult to feel amusement and embarrassment at the same time.

\section{Vi. Smiles Machine: Automatic generation of VIRTUAL SMILING FACES}

Based on the unsupervised symbolic analysis, we have developed procedures to automatically reconstitute a virtual smiling face given a point in a PCA plane. In Section A, we first present the procedure to automatically compute the characteristics of a virtual smiling face (i.e., the histogram of frequencies of the variables describing the smiles) from a PCA point in the plan. Then, in Section B, we present a procedure to generate the smiling virtual face from these characteristics.

\section{A. Reconstitution of virtual smile's characteristics from a PCA point}

Let $u_{j}$ be the unit vector of the $j^{\text {th }}$ factor among $R$ of a PCA class of smiles such that $u_{j}^{T}=\left(u_{j_{1}}, \ldots, u_{j_{R}}\right)$ where $R=\sum_{l=1}^{p} m_{l}$ where $m_{l}$ is the number of modalities (i.e., possible discrete values) of the variable $l$ among the $p$ symbolic variables. For more technical details on this PCA, see [28]. We have by definition $u_{j}^{T} u_{j}=1$. It is known that:

$$
\sum_{j=1}^{R} u_{j} u_{j}^{T}=1_{R}
$$




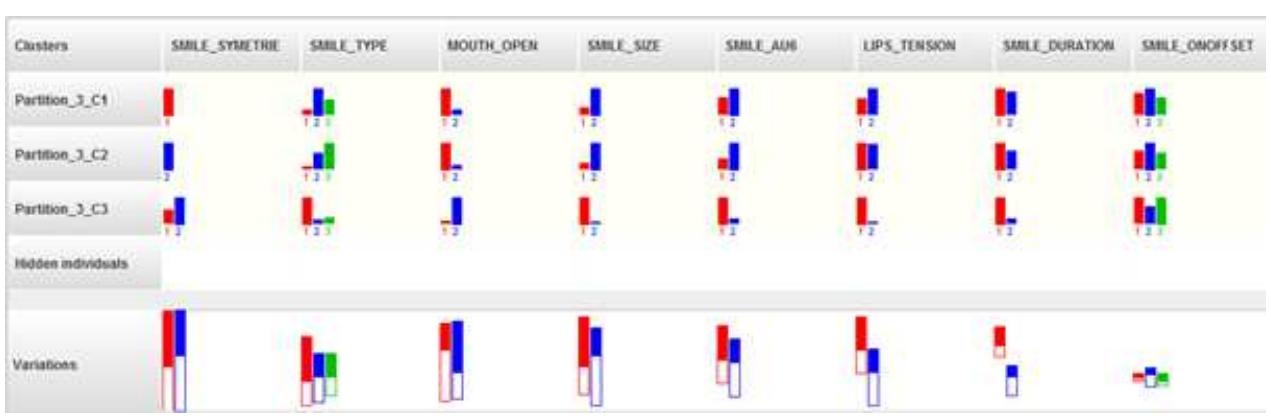

Fig. 6. Variables of the 3 classes of smile obtained by an unsupervised classification.

where $1_{R}$ is the identity matrix of $\mathrm{R}$ dimension.

Let $\mathrm{v}$ be a point defined by the vector $\mathrm{W}=\left(w_{1}, \ldots, w_{R}\right)$ in the initial normalized (i.e, centered and reduced) space. The projection of $\mathrm{v}$ in the factor space is defined by $V$ a vector such as $V^{T}=\left(v_{1}, \ldots, v_{R}\right)$ where $v_{j}$ is the coordinate of the projection of $v$ on the factor axis $u_{j}$. Therefore, we have: $W^{T} u_{j}=v_{j}$ which implies $W^{T} u_{j} u_{j}^{T}=v_{j} u_{j}^{T}$.

From (1), we obtain $W^{T}=\sum_{j=1}^{p} v_{j} u_{j}^{T}$, which can be written:

$$
W^{T}=\left(\sum_{j=1}^{p} v_{j} u_{j 1}, \ldots, \sum_{j=1}^{p} v_{j} u_{j R}\right) .
$$

In order to reconstitute the smile corresponding to the $v$ point, we denote its coordinates in the initial space by $X^{T}=$ $\left(x_{1}, \ldots, x_{R}\right)$. The link between $V$ and $X$ is given by:

$$
W^{T}=\left(\left(x_{1}-\mu_{1}\right) / \sigma_{1}, \ldots,\left(x_{R}-\mu_{R}\right) / \sigma_{R}\right)
$$

where $\mu_{k}$ and $\sigma_{k}$ are, respectively, the mean and the mean square of the $j^{t h}$ variable in the case of a PCA of single smile and the mean and mean square of frequencies of categories in the case of a PCA of classes of smiles. It follows from (2) and (3) that: $\left(x_{k}-\mu_{k}\right) / \sigma_{k}=\sum_{j=1}^{R} v_{j} u_{j k}^{T}$. Therefore, we can reconstitute the coordinates of the smile associated with the point $v$ of the factorial plane by:

$$
x_{k}=\mu_{k}+\sigma_{k} \sum_{j=1}^{R} v_{j} u_{j k}^{T} .
$$

In practice, in the graph of the principal components, we can imagine the user clicking on a point in the graph and moving in the first plane defined by the two axis of the largest eigen value. An approximation of the smile reconstitution can be obtained by taking care on only the two first (or more) factorial axes of largest eigen value by replacing $\mathrm{R}$ by 2 (or more), in the formula (4). The user can improve the smile (reconstituted by its coordinates defined by (4) or its approximation), by using other factorial planes, for example the third and following axes crossed with the first axis, until he get the desired smiling face

The procedure presented in this section enables us to easily obtained from a point in the PCA plane the histogram of each variable describing the smile corresponding to this point. We use these data to reconstitute the virtual smiling face. We present the procedure in the next section.

\section{B. Reconstitution of a virtual smiling face}

Based on the histograms resulting from the procedure presented above, we have developed a procedure to automatically reconstitute a smiling virtual face corresponding to a point in the PCA plane. The procedure is based on a deformation of the prototypical expressions of amused, polite and embarrassed smiles (Figure 3). These prototypical smiling faces have been designed manually by manipulating facial muscles. The deformations of the facial muscles are represented with a set of values corresponding to facial animation parameters (FAPs) of the MPEG-4 standard [29]). Given the facial muscles implied in an expression of a smile, each prototypical smiling face is represented by 25 values of FAPS, corresponding to the deformation of 25 facial muscles.

To reconstitute a smiling virtual face of a particular point of the PCA plane, we consider the histograms of the 7 variables of this point (openness of the mouth, lips tension, smile size, symmetry of the smile, cheek raising, duration of the onset and offset and total duration of the smile). These histograms are obtained following the procedure described in the previous section (Section A).

The procedure to reconstitute the smiling face from these histograms is based on a weighting of the values of the FAPS with the frequencies of the histograms of the variables. The variables corresponding to the openness of the mouth, the lips tension, the smile size, the symmetry of the smile, and the cheek raising are described with particular values of FAPS. For instance, the cheek raising (SMILE_AU6) is characterized by the FAPS 21, 22, 39, 40, 41, and 42. Consequently, with each modality of these variables can be associated a set of values of FAPS. For instance, the FAPS values for the modalities AU6 and no_AU6 of the variable SMILE_AU6 are described in Table I.

\begin{tabular}{|c|c|c|}
\hline FAPS & AU6 & no_AU6 \\
\hline 21 & 100 & -300 \\
\hline 22 & 100 & -300 \\
\hline 39 & -100 & 0 \\
\hline 40 & -100 & 0 \\
\hline 41 & 400 & -100 \\
\hline 42 & 400 & -100 \\
\hline \multicolumn{3}{|c|}{ TABLE I }
\end{tabular}

VALUES OF FAPS FOR THE SMILE_AU6 VARIABLE

The problem is that some modalities have FAPS in common. For instance, the lips tension is described by 19 FAPS among 
which 13 are also used to describe the openness of the mouth. Consequently, we cannot apply a simple weighting of the FAPS of the modality with the highest frequency of the histogram. To overcome this problem, we have considered two types of variables: the variables that define the value of the FAPS (i.e., MOUTH_OPEN and SMILE_SIZE) and the variables that modulate these values (i.e., SMILE_SYM and LIPS_TENSION). Based on the analysis of the FAPS of the prototypical smiles, we have defined vectors of values of FAPS corresponding to the following modalities: small open mouth, large open mouth, small size, large size, AU6 and no-AU6. Moreover, we have defined the deformation that the asymmetry and the tension of the lips generate on the FAPS by weights for each FAPS implied for these variables. For instance, the asymmetry may be modeled by a vector of 5 weights corresponding to the weighting to perform on the 5 FAPS to simulate asymmetry ${ }^{5}$. Finally, given the histograms of a class as input, the following procedure is performed:

1) for each variable MOUTH_OPEN and SMILE_SIZE: select the modality of the variable with the highest frequency in the histogram;

2) select the corresponding values of FAPS;

3) weight the values of the FAPS related to MOUTH_OPEN and SMILE_SIZE with the corresponding frequency extracted from the histogram of the variable;

4) weight the values of the FAPS related to SMILE_SYM and LIPS_TENSION using the weights themselves weighted by the frequency of the lips tension and asymmetry modalities described in the histogram.

This procedure enables us to generate different combinations of smiles. We illustrate the results of the procedure on the partition in Figure 8. Note that this PCA differs from the PCA in Figure 7 since we have added 2 partitions of 3 classes $^{6}$ and we have considered the TYPE_SMILE variable additionally to the 7 variables. In Figure 8, the smiling virtual faces of the classes have been generated using the procedures presented above. The correlation circles corresponding to this PCA are represented in Figure 9. On the correlation circles, the modalities of the variable SMILE_TYPE highlight the characteristics of the three smiles (amused, embarrassed and polite) and their position in the plane 8 . The correlation circle shows that the axis can correspond to a "smile multidimensional space" going from the smiles the most embarrassed (lower left part) to the smiles the most amused (right part), the polite smiles being rather at the top.

\section{CONCLUSION}

In conclusion, in this paper, we have presented a method to automatically generate smiling virtual faces. This method

\footnotetext{
${ }^{5}$ For the lips tension, given that the deformation of the FAPS resulting from the tension of the lips depends on the size and openness of the mouth, we have defined vectors of weights for the following combinations of modalities: large size open mouth, large size close mouth, small size open mouth and small size close mouth.

${ }^{6}$ one reflecting the prototypical smiling faces: Part $3 \mathrm{C} 1$, Part $3 \mathrm{C} 2$, and Part $3 \mathrm{C} 3$, and one corresponding to the unsupervised analysis in 3 classes illustrated in Figure 6: Part $3 \mathrm{C} 1$ clust, Part $3 \mathrm{C} 2$ clust, and Part $3 \mathrm{C} 3$ clust.
}

is based on an unsupervised symbolic analysis of a corpus of virtual smiling faces directly created by users. The method makes it possible to generate different types of smiles of amusement, politeness and embarrassment but also combinations of these smiles by simply selecting a point (or a class) in a multidimensional space (PCA plane).

The originality of the proposed method comes from different aspects. First of all, most of the virtual characters express one or at most two different smiles during an interaction with a user. In this paper, we present a method to increase considerably the repertoire of a virtual character's smiling expressions. Moreover, the approach generally used for the synthesis of facial expressions is based on empirical and theoretical research in human and social sciences describing the characteristics of human facial expressions. In this article, the synthesis of facial expressions is based on a corpus of virtual smiling faces directly created by the users. Secondly, some previous research works have proposed models to generate automatically facial expressions from a point in a dimensional space. However, the latter are generally based on classical numerical data analysis and do not enable the generation of combinations of different types of smiles.

In the next step of this work, we aim at analyzing the perception of the users of the generating smiling faces to evaluate if the combinations of meanings are perceived through the smile expressions.

\section{ACKNOWLEDGMENT}

This research has been supported by the European Community Seventh Framework Program (FP7/2007- 2013), under grant agreement no. 231287 (SSPNet). 


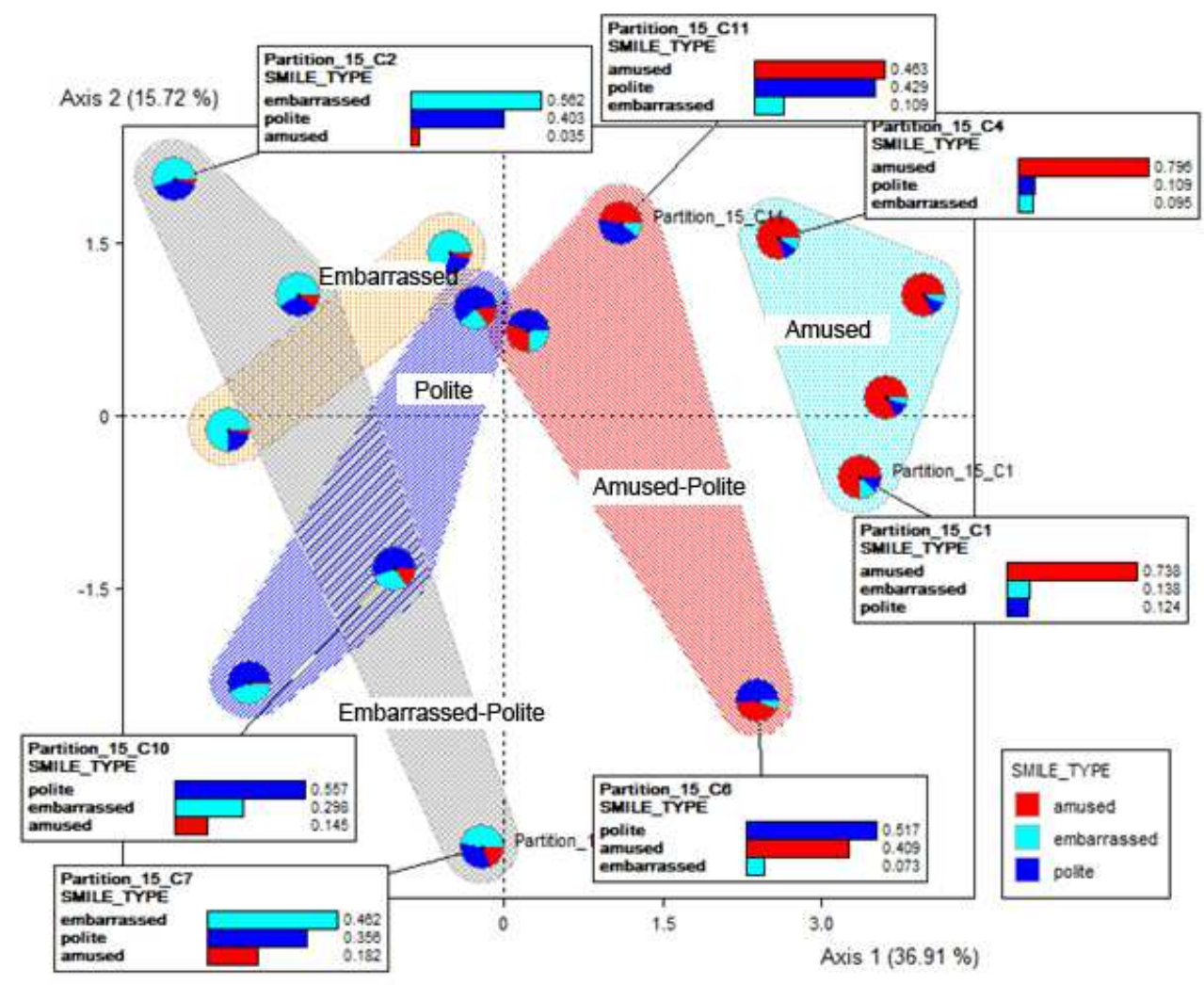

Fig. 7. Typology in 5 classes based only on the type of smile variable (SMILE_TYPE). Note that even if an overlap of the clusters appears in the figure, there is no overlap of the classes.

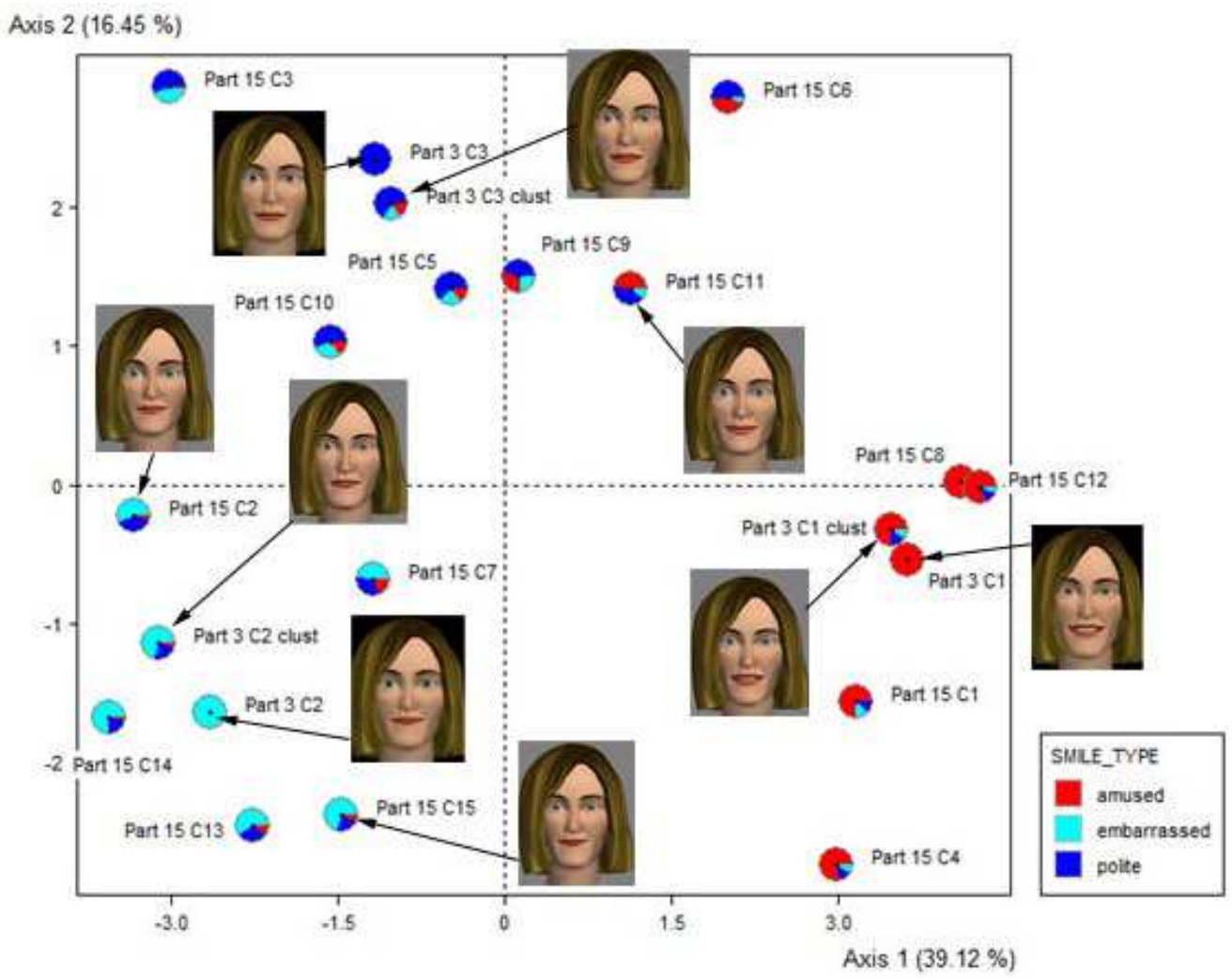

Fig. 8. Automatically generated smiling faces. 

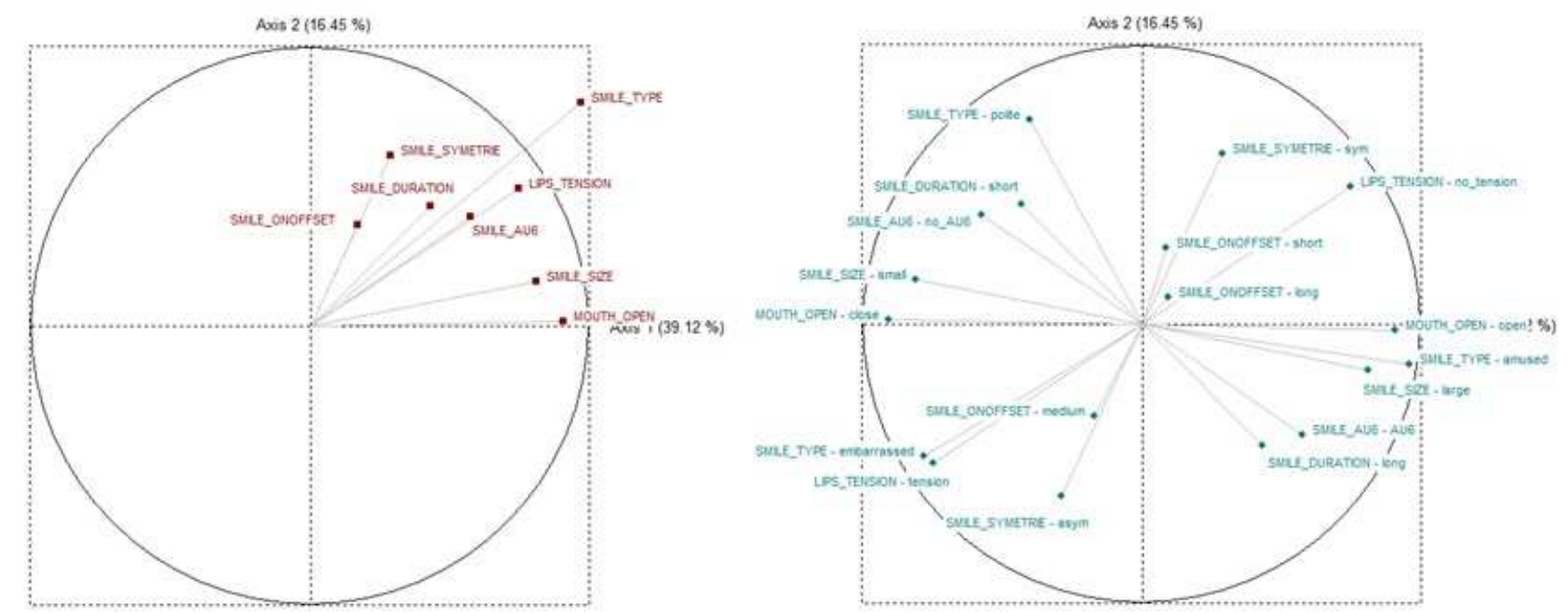

Fig. 9. Correlation circle of the symbolic variables (left) and of the modalities of the variables (right). 


\section{REFERENCES}

[1] J. Cassell, "More than just another pretty face: Embodied conversational interface agents," Communications of the ACM, vol. 43, pp. 70-78, 2000.

[2] N. C. Krämer, "Social effects of virtual assistants. a review of empirical results with regard to communication," in Proceedings of the 8th international conference on Intelligent Virtual Agents. Berlin, Heidelberg: Springer-Verlag, 2008, pp. 507-508.

[3] M. Knapp and J. Hall, Nonverbal Communication in Human Interaction. Wadsworth Publishing, 2009.

[4] S. Lau, "The effect of smiling on person perception," Journal of Social Psychology, vol. 117, pp. 63-67, 1982.

[5] E. Krumhuber, A. Manstead, D. Cosker, D. Marshall, and P. Rosin, "Effects of Dynamic Attributes of Smiles in Human and Synthetic Faces: A Simulated Job Interview Setting," Journal of Nonverbal Behavior, vol. 33, pp. 1-15, 2008.

[6] G. Theonas, D. Hobbs, and D. Rigas, "Employing Virtual Lecturers' Facial Expressions in Virtual Educational Environments," International Journal of Virtual Reality, vol. 7, pp. 31-44, 2008.

[7] B. Leite Dantas Bezerra and F. A. Tenorio de Carvalho, "Symbolic data analysis tools for recommendation systems," Knowledge and Information Systems, vol. 26, no. 3, pp. 385-418, 2011.

[8] C. Quantin, L. Billard, M. Touati, N. Andreu, Y. Cottin, M. Zeller, F. Afonso, G. Battaglia, D. Seck, G. LeTeuff, and E. Diday, "Classification and regression trees on aggregate data modeling: An application in acute myocardial infarction," Journal of Probability and Statistics, vol. 2011, 2011.

[9] C. Fablet, E. Diday, S. Bougeard, C. Toque, and L. Billard, "Classification of Hierarchical-Structured Data with Symbolic Analysis. Application to Veterinary Epidemiology," in International Conference on Computational Statistics, 2010.

[10] R. Haddad, F. Afonso, and E. Diday, "Approche symbolique pour l'extraction de thmatiques: Application un corpus issu d'appels tlphoniques," in Proceedings of "Rencontres de la Societe francophone de Classification”, 2011.

[11] I. Albrecht, M. Schroder, J. Haber, and H. Seidel, "Mixed feelings : expression of non-basic emotions in a muscle-based talking head," Special issue of Journal of Virtual Reality on Language, Speech and Gesture, vol. 8(4), pp. 201-212, 2005.

[12] T. D. Bui, "Emotions And Facial Expressions For Embodied Agents," Ph.D. dissertation, University of Twente, Department of Computer Science, 2004.

[13] H. Maldonado, J. Lee, S. Brave, C. Nass, H. Nakajima, R. Yamada, K. Iwamura, and Y. Morishima, "We learn better together: enhancing elearning with emotional characters," in Computer Supported Collaborative Learning 2005: The Next 10 Years!, T. Koschmann, D. Suthers, and T. Chan, Eds. Lawrence Erlbaum Associates, Mahwah, NJ, 2004, pp. 408-417.

[14] R. Beale and C. Creed, "Affective interaction: How emotional agents affect users," International Journal of Human-Computer Studies, vol. 67, no. 9, pp. 755-776, 2009.

[15] P. Ekman and W. V. Friesen, Unmasking the Face. A guide to recognizing emotions from facial clues. New Jersey: Prentice-Hall, Inc., Englewood Cliffs, 1975.

[16] Z. Ruttkay, H. Noot, and P. T. Hagen, "Emotion disc and emotion squares: tools to explore the facial expression face," Computer Graphics Forum, vol. 22, no. 1, pp. 49-53, 2003.

[17] P. Ekman, Telling Lies: Clues to Deceit in the Marketplace, Politics, and Marriage, ww norton \& co ed., 2009.

[18] E. Krumhuber and A. Manstead, "Can Duchenne smiles be feigned? New evidence on felt and false smiles," Emotion, vol. 9, no. 6, pp. 807-820, 2009.

[19] Z. Ambadar, J. F. Cohn, and L. I. Reed, "All Smiles are Not Created Equal: Morphology and Timing of Smiles Perceived as Amused, Polite, and Embarrassed/Nervous," Journal of Nonverbal Behavior, vol. 17-34, pp. 238-252, 2009.

[20] M. Ochs, R. Niewiadomski, P. Brunet, and C. Pelachaud, "Smiling virtual agent in social context," Cognitive Processing, Special Issue on "Social Agents", 2011.

[21] E. Diday and L. Billard, "Symbolic Data Analysis : Conceptual statistics and data Mining," in Symbolic Data Analysis, ser. Wiley Series in Computational Statistics, D. E. . B. L., Ed. Wiley Interscience, 2006, pp. $1-321$.

[22] E. Diday, Handbook of categorization in cognitive science, 2005, ch. Categorization in Symbolic Data Analysis.

[23] M. Noirhomme-Fraiture and E. Diday, Symbolic Data Analysis and the SODAS Software. Wiley Interscience, 2008.
[24] H. Cohen and C. Lefebvre, Handbook of Categorization in Cognitive Science, 1st Edition. Elsevier, 2005.

[25] M. Noirhomme-Fraiture and M. Rouard, Analysis of Symbolic Data, 2000, ch. Visualizing and Editing Symbolic Objects.

[26] G. Milligan and M. Cooper, "An examination of procedures for determining the number of clusters in a data set," Psychometrika, vol. 50, no. 2, pp. 159-179, 1985.

[27] E. Diday and M. Noirhomme-Fraiture, Validation of Clustering Structure: Determination of the Number of Clusters. John Wiley and Sons, Ltd, 2008, pp. 235-262.

[28] E. Diday, "Principal component analysis for bar charts and metabins tables," Statistical Analysis and Data Mining, vol. 6-5, pp. 403-430, 2013.

[29] J. Ostermann, "Face animation in mpeg-4," in MPEG-4 Facial Animation - The Standard Implementation and Applications, I. Pandzic and R. Forchheimer, Eds. Wiley, England, 2002, pp. 17-55. 